\title{
The influence of milling technology on the crystallite size and granulometry of tricalcium aluminate
}

\author{
Simona Ravaszová ${ }^{1}$, Karel Dvořák ${ }^{2}$ \\ Faculty of Civil Engineering, Brno University of Technology, Brno, Czech Republic \\ E-mail: ${ }^{1}$ ravaszova.s@fce.vutbr.cz (correspondingauthor)
}

\begin{abstract}
The article deals with the monitoring and evaluating of the length of the milling process on the crystallographic and granulometric properties of the cubic tricalcium aluminate. Tricalcium aluminate was prepared by solidstate reaction and subjected to milling in a dry and wet conditions of three laboratory mills. The evaluation of the influence of the milling technology on the crystallite size was carried out on the selected main three crystallographic planes. The calculation of the crystallite size was based on the full width of half maximum. Full width of half maximum has been calculated for all the above diffraction lines in the HighScore Plus program. All types of selected laboratory mills in both dry and wet conditions for all three crystallographic planes affect crystallite size of tricalcium aluminate. In terms of granulometry, the material was very effective and significantly refined. The problem arises with long grinding time, where the material tended to form agglomerates.
\end{abstract}

Keywords: tricalcium aluminate, milling, X-Ray powder diffraction, full width of half maximum, Scherrer equation.

\section{Introduction}

Portland cement is a mixture of the clinker phases alite, belite, aluminate, ferrite, calcium sulfate and other minor constituents. Some properties of cement are not only influenced by the proportion of the individual clinker phases but also by the composition of the phases themselves, because the clinker phases are not pure compounds, but form solid solutions with other oxides in the clinker (Stephan, \& Wistuba, 2006; Staněk, 2016).

$\mathrm{C}_{3} \mathrm{~A}$ in an impure form is one of the main components of Portland cement. Production clinkers have been found to contain cubic or orthorhombic forms of aluminate, alone or in combination. The monoclinic modification has not been observed. The orthorhombic modification is also known as the prismatic, dark intersitial material, and is sometimes pseudotetragonal. It can arise only if sufficient alkali is available, but its formation appears to be favoured also by rapid cooling and by bulk compositions potentially able to yield a relatively high proportion of aluminate Cubic aluminate in clinker is often finely grained and closely admixed with dendritic crystals of ferrite. The XRD powder pattern is characterized by strong, singlet peaksat approximately $33.3^{\circ}, 47.7^{\circ}$ and $59.4^{\circ} 2 \theta$ (CuK $\alpha$ radiation), whose indices are, respectively 400, 800 and 844 (Duda, 1988). The cubic form of $\mathrm{C}_{3} \mathrm{~A}$ is normally encountered in technical clinkers, but if sodium concentrations in the clinker are high enough, it is common that part of the $\mathrm{C}_{3} \mathrm{~A}$ occur in the orthorhombic modification. The cubic $\mathrm{C}_{3} \mathrm{~A}$ can be easily sintered without any need for stabilization (Taylor, 1997; Maki, 1973). Pure $\mathrm{C}_{3} \mathrm{~A}$ contains $62.3 \% \mathrm{CaO}$ and $37.7 \% \mathrm{Al}_{2} \mathrm{O}_{3}$. Substantial proportions of both $\mathrm{Ca}$ and $\mathrm{Al}$ are thus replaced, the total content of substituent oxides being typically around $13 \%$ for the cubic modification. The content of equivalent $\mathrm{Na} 2 \mathrm{O}$ appears to be around 1\% (Staněk, Sulovský, \& Boháč, 2017; Hewlett, 2003).

The laboratory mills are divided according to the milling principle. The high-speed planetary mill is used wherever a high degree of fineness is required. This type of mill utilizes the principle of centrifugal acceleration, milling by friction and impact, and produces high mechanical activation at relatively low milling times. The disc vibratory mill grinds friction and produces analytical fineness of the material at a very fast time. McCrone micronising mill is a special type of mill that is designed to prepare samples for X-Ray analysis. With the growing time of milling, the material gets so refined that agglomeration and aggregation may occur, which means that when the material is grinded, the individual particles are distributed but subsequently very compressed. There is an electrostatic charging of the particle surface with opposite charges (Ravaszová \& Dvořák, 2018). 


\section{Materials and methods}

The modified Wesselsky-Jensen method (Wesselsky \& Jensen, 2009) was used to prepare cubic tricalcium aluminate. Calcium carbonate $\mathrm{CaCO}_{3}$ (p.a. 99.8\%) and aluminum oxide $\mathrm{Al}_{2} \mathrm{O}_{3}$ (p.a. 99.0\%) were used to prepare a tricalcium aluminate. The raw materials were dosed in an amount that corresponds to the molar ratio given in the WesselskyJensen method. The raw materials mixture with water were homogenized in the PULVERISETTE 6 planetary mill at $500 \mathrm{rpm}$ for 3 minutes. The suspension after wet milling was dried in laboratory oven at $105{ }^{\circ} \mathrm{C}$ for 24 hours. The platinum crucibles were filled into $3 / 4$ volume of dried raw material mixture and compacted manually. Ventricular high temperature kiln with superkanthal heating elements was used for solid state reaction. Solid state reacton was carried out at temperature of $1450{ }^{\circ} \mathrm{C}$ with 3 hours of soaking on accordance with the Wesselsky-Jensen method. At the end of firing mode, the platinum crucibles were removed from the furnace and immediately cooled down with air flow.

Phase composition control was performed by X-ray analysis on Panalytical Empyrean XRD with $\mathrm{Cu}-\mathrm{K} \alpha$ as the radiation source, $\lambda=1,540598$ for $\mathrm{K} \alpha 1$, accelerating voltage $45 \mathrm{kV}$, beam current $40 \mathrm{Ma}$, diffraction angle $2 \theta$ in the range from $5^{\circ}$ to $80^{\circ}$ with a step scan of $0.01^{\circ}$.

Tricalcium aluminate was adjusted to an entry specific surface area of $290 \mathrm{~m}^{2} / \mathrm{kg}$. The material was milled in a Fritsch Pulverisette 6 planetary mill to achieve a specific surface area. Blaine specific surface area was measured using a PC-Blaine-Star automatic device with a measurement cell capacity of 7.95 cubic centimeters. The measurement was performed three times to eliminate errors and the resultant value was the average of three readings.

The main aim of this article is monitoring of the milling process of tricalcium aluminate in three types of laboratory mills. At the same time, the material was subjected to milling in dry and wet conditions. Isopropyl alcohol was used as an inert liquid for wet milling. The milling was done using the PULVERISETTE 6 planetary mill, the RS 200 vibratory disk mill from RETSCH and the McCrone Micronising mill (Figure 1). Fritsch planetary ball mill Pulverisette 6 in a bowl with the volume of $0.5 \mathrm{dm}^{3}$ was used. The bowl was filled by 25 steel milling balls with $20 \mathrm{~mm}$ in diameter. The comminution of the material takes place primarily through the high-energy impact of the milling balls. The milling bowl, containing the milling balls and the material to be ground, rotates around its own axis on the main disk whilst rotating rapidly in the opposite direction. Centrifugal force causes the 3 ground sample material and the milling balls to separate from the inner wall of the milling bowl. The milling balls then cross the bowl at high speed and further grind the sample material by impact against the opposite wall. The Vibratory Disc Mill RS 200 comminutes by impact and friction. The milling set is firmly attached to the vibration plate with a quick-action lever. The plate with the milling set is subjected to circular horizontal vibrations. The centrifugal force acting on the milling rings in the dish results in extreme pressure. Impact and frictional forces acting on the sample produced analytical fineness in 1-3 minutes. The circular vibrations are produced by a frequency controlled $1.5 \mathrm{~kW} 3$-phase motor. McCrone Micronising Mill is designed only for wet milling. 5 periods of milling were set at 1, 5, 30, 60 and 120 minutes. The milling in the planetary mill was set at the optimum speed for the tricalcium aluminate $300 \mathrm{rpm}$ and in a vibratory disk mill at $1200 \mathrm{rpm}$. The particle distribution and fineness were determined using the Malvern Mastersizer 2000 laser granulometer.

The crystallites size was evaluated on selected diffractive lines at appropriately selected crystallographic planes that had a major crystallographic direction. Three distinct crystallographic planes were selected. They are determined by the Miller Index "hkl": 800, 440, 844.

Calculation of the size of crystallites was based on the measurement of full width of half maximum (FWHM). The measurement of FWHM was carried out using the HIGHSCORE PLUS program. The FWHM values were obtained in the program HIGHSCORE PLUS for selected diffraction lines. From the list of peaks and their FWHM, the data for the lines that responded to the $\mathrm{K} \alpha 2$ radiation were deleted.

FWHM includes a size-strain, that has been evaluated by HighScore Plus program for a dry milling in planetary mill PULVERISETTE 6. The size-strain at the beginning of the milling and the end was the same, so Williams-Hall plots were droped and the size of the crystallite was evaluated only by the Scherrer Eq. (1) (Balaz, 2008):

$$
B(2 \theta)=\frac{K \cdot \lambda}{L \cdot \cos \theta}
$$

for the $\lambda=1.540598$ for the $C u$ cathode and the Scherrer constant $K=0.94$, crystallite size values were obtained without device extension.

The device extension was set to the $\mathrm{LaB}_{6}$ standard (lanthanum hexaborite). $\mathrm{LaB}_{6}$ is considered to be a fully crystalline material in which the size of crystallites is theoretically equal to infinity. Diffraction lines of LaB 6 were determined in the same positions as the selected diffraction lines in the same way in the HIGHSCORE PLUS program.

Using the modified Scherrer equation, the crystallites size was obtained according to the Eq. (2):

$$
L=\frac{K . \lambda}{\cos \theta} \cdot \frac{1}{\beta}=\frac{K . \lambda}{\cos \theta} \cdot \frac{1}{\sqrt{B^{2}-b^{2}}}
$$

$L$ is crystallite size, $K$ is the Scherrer constant, $\lambda$ is wavelength of X-ray radiation, $\theta$ is diffraction angle, $B$ is FWHM, $b$ is FWHM of used standard $\left(\mathrm{LaB}_{6}\right)$. 

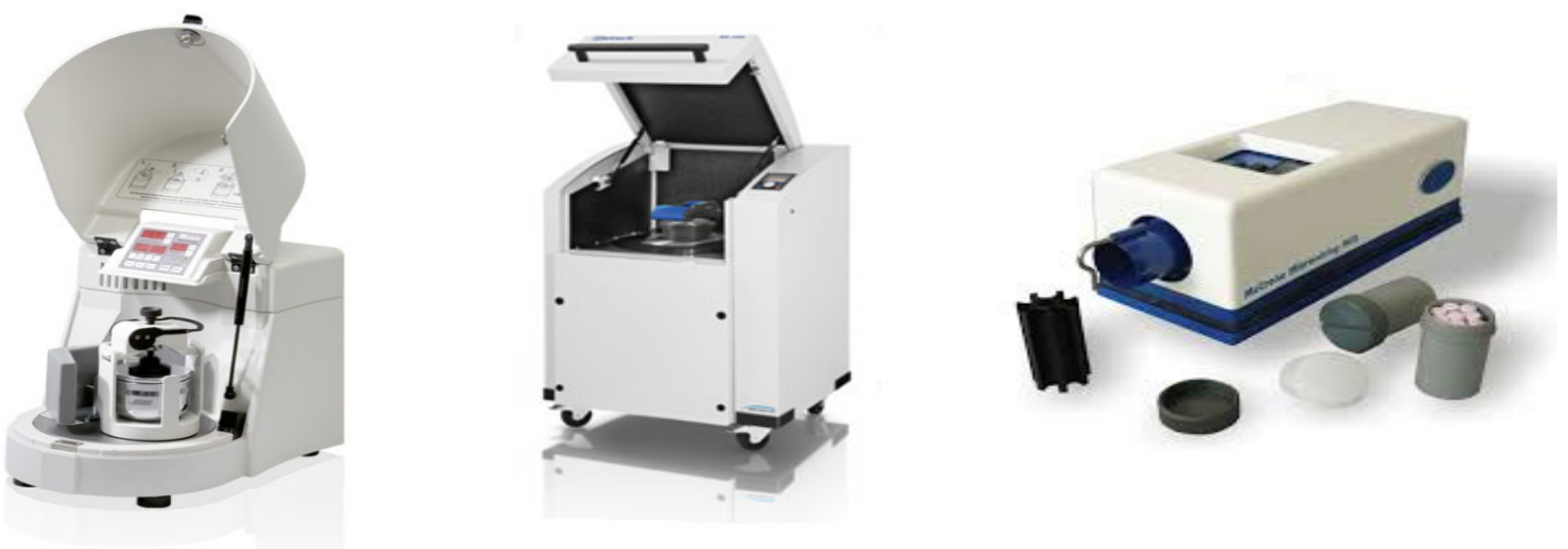

Figure 1. PULVERISETTE 6 planetary mill, RS 200 vibratory disk mill and McCrone Micronising mill

\section{Results}

The quality control of the mineralogical composition was performed by X-Ray analysis, the scan of which is shown in Figure 2 .

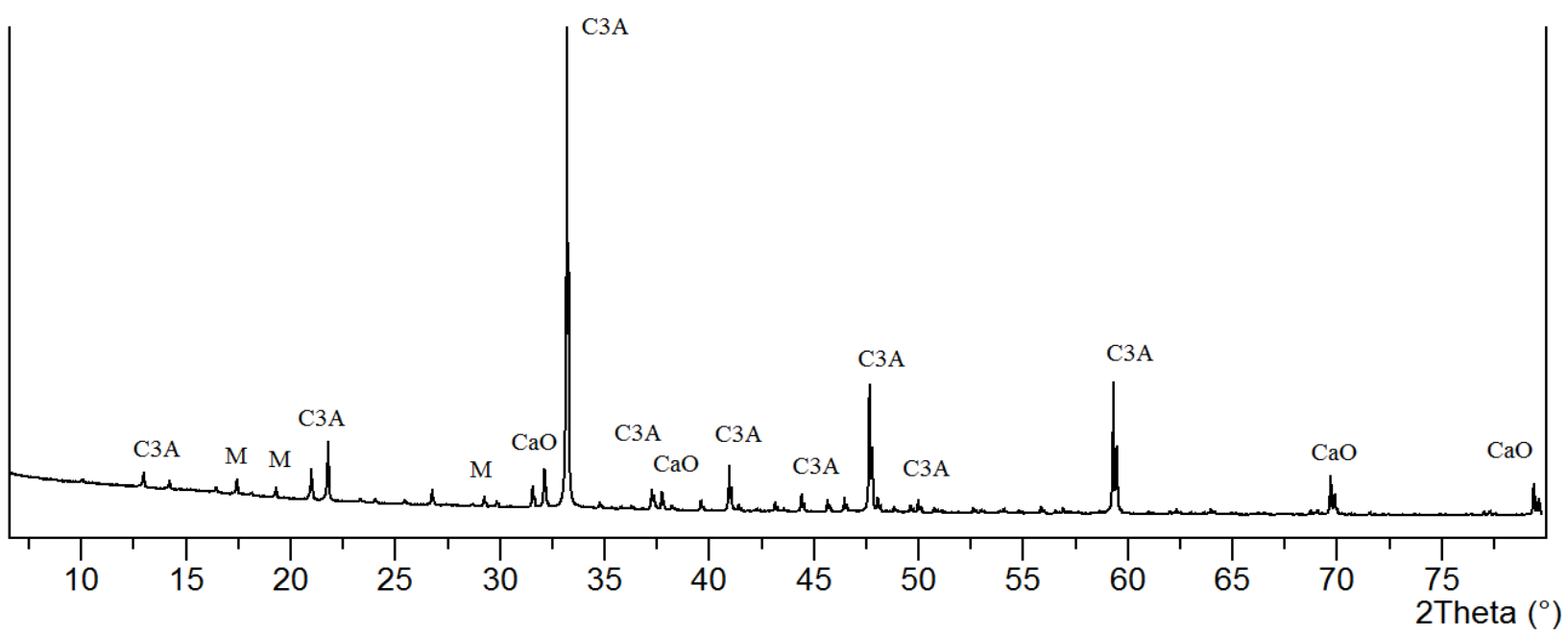

Figure 2. X-Ray diffraction patterns of tricalcium aluminate ( $\mathrm{C}_{3} \mathrm{~A}$-cubic, $\mathrm{CaO}$-free lime, $\mathrm{M}$-mayenit)

The main identifiable mineral phase was cubic tricalcium aluminate. Furthermore, minor phases of corundum and free lime appeared. Nevertheless, the major crystal phase in the samples is tricalcium aluminate which was successfully synthetized from the designed mixture. It was adjusted in a vibratory mill to a specific surface area of $290 \mathrm{~kg} / \mathrm{m}^{2}$ according to the Blaine method.

The effect of milling technology on clinker mineral crystallinity was evaluated by changing the shape and FWHM. For better clarity, a graph for individual laboratory mills and milling technology was prepared only at 1 and 120 minutes for crystallograpic plane 440 (Figure 3).

It can be seen on the diffraction patterns that the 1 minute of milling technology has almost no impact on peak shape deformation. At 120 minutes the deformation manifests most. Wet milling due to the presence of isopropyl alcohol liquid did not have such a drastic impact on the peak shape as a dry milling. The greatest change in the shape of the peaks was seen in the dry milling of both the vibratory mill, where the greatest deformation can be observed, as well as in the planetary mill. It can be seen on the diffraction pattern that the peaks were reduced and broadened.

It is clear from the graphs on Figure 4 that in all cases up to the crystallite size of $2000 \AA$ the contribution of device extension is constant. Above the $2000 \AA$ crystallite size, the device extension is almost double and affects the outcome. This corresponds to the applicability of the method to a crystallite size of $200 \mathrm{~nm}$, which corresponds to $2000 \AA$.

In the following graphs, the effect of milling on the size of crystallites in the crystallographic planes 400,800 and 844 is shown (Figure 5). 

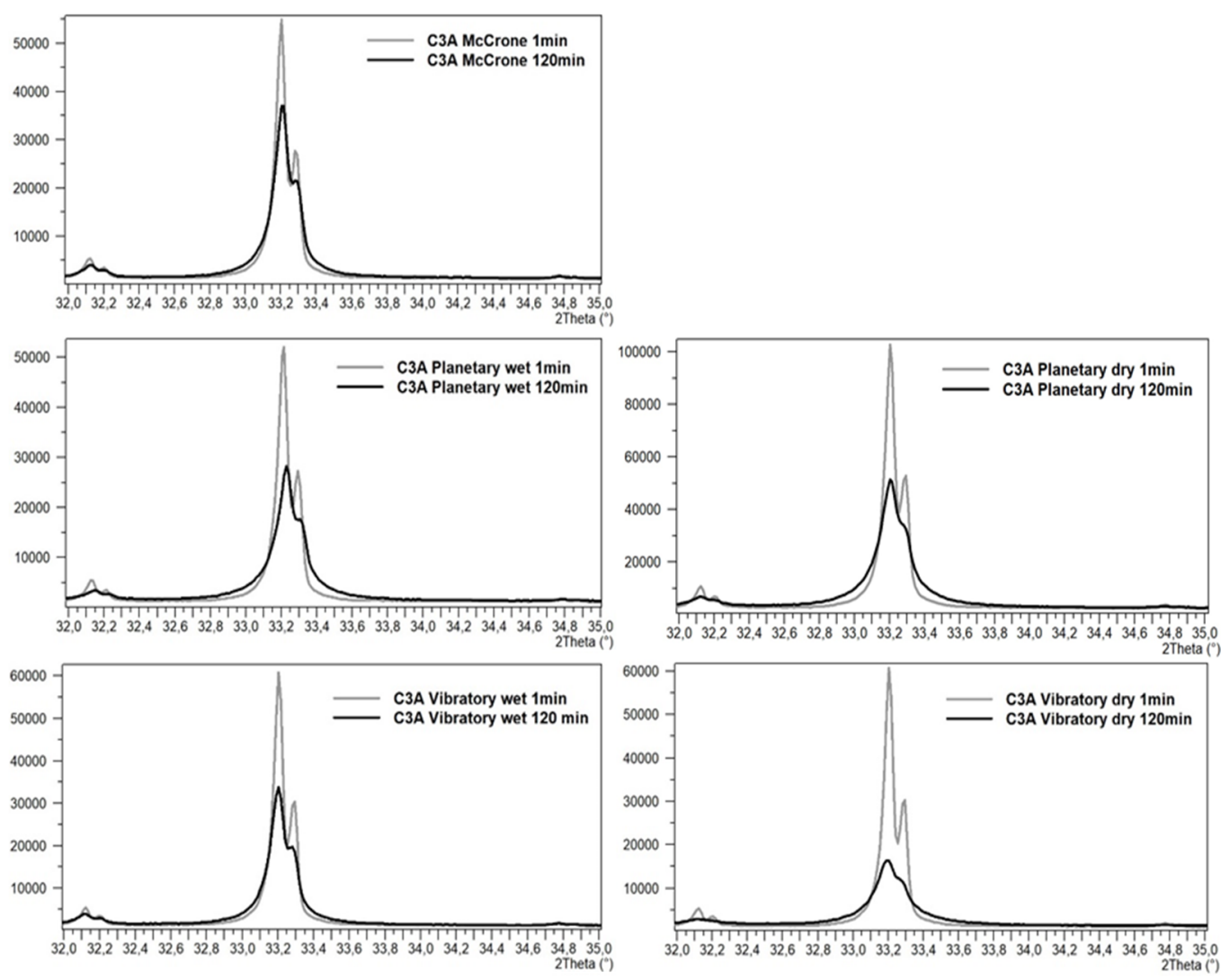

Figure 3. The impact of milling technology on the shape and FWHM in the crystallographic plane 440
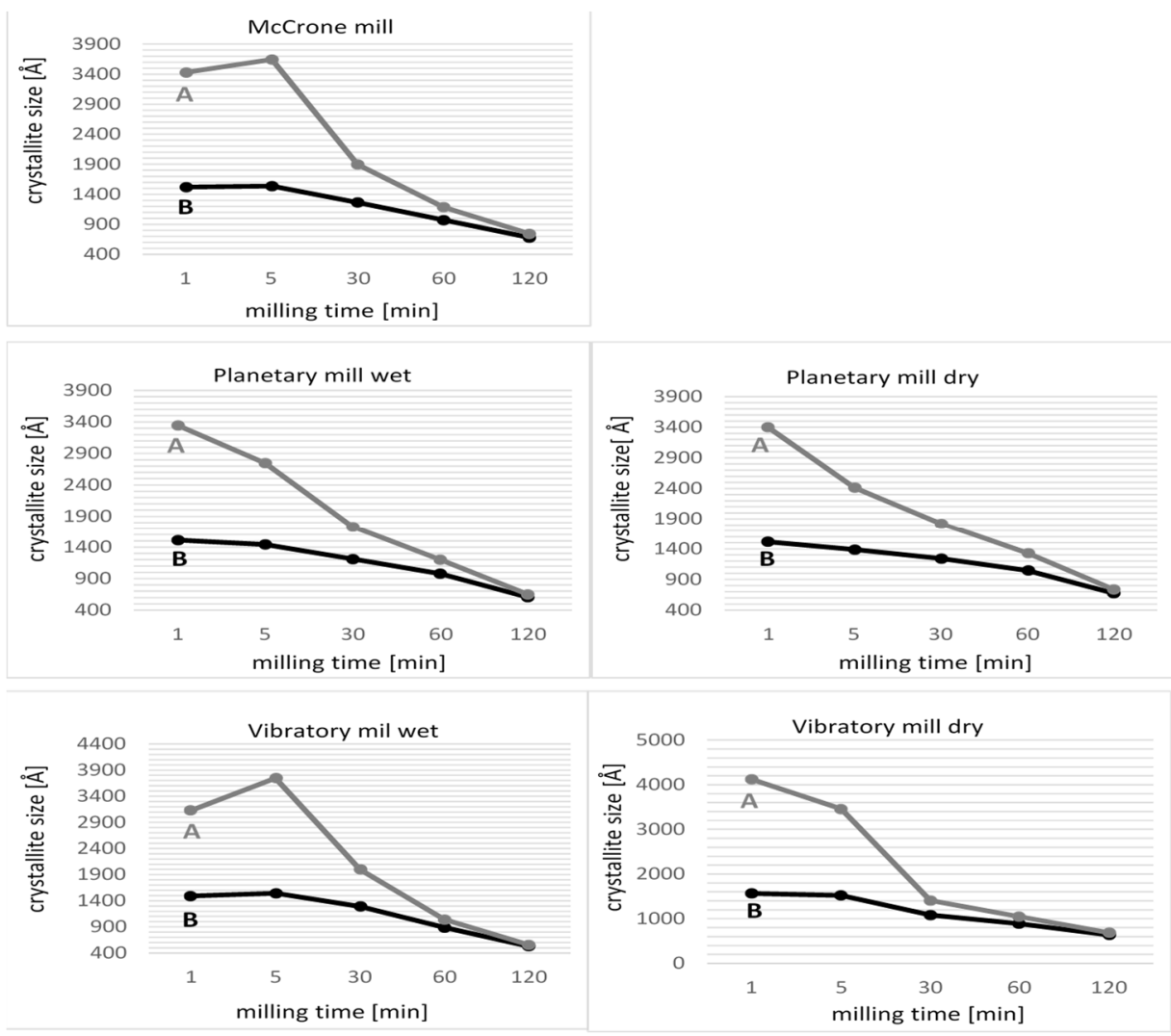

Figure 4. Contribution of instrument extension on crystallite size before and after correction in crystalographic plane 440 (A-after, B-before) 

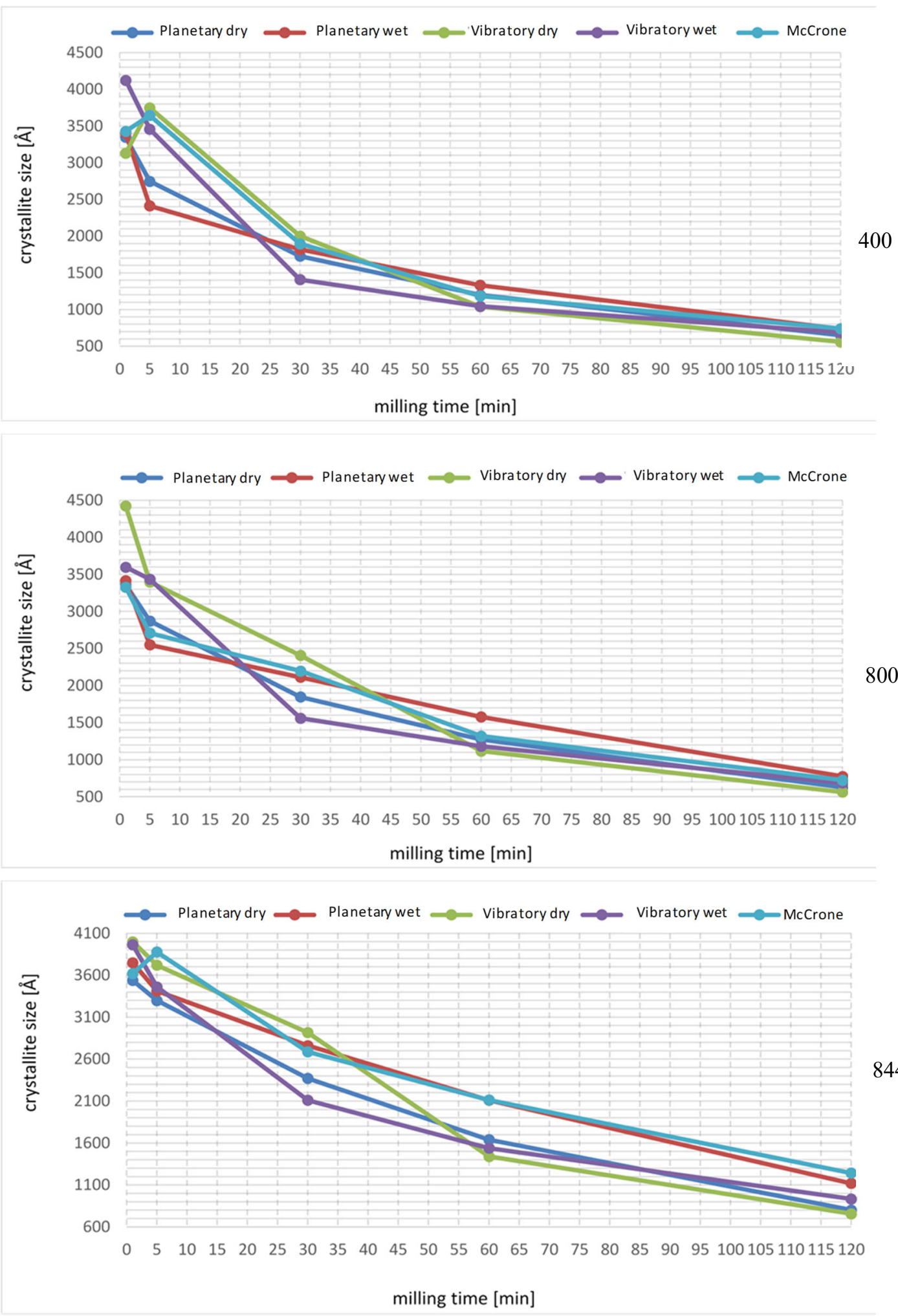

Figure 5. Graphical comparison of crystallite size at milling time for all types of laboratory mill and technologies

All types of used laboratory mills, both in dry and wet conditions, affect the size of crystallites. The trend of changing the size of the crystallites is similar in all selected crystallographic planes. The most significant reduction in the size of the crystallites occurs during dry milling in a planetary and vibratory mill.

On the Figure 6 is a graph of particle sizes where all types of mills and milling technology are compared at 120 minutes. 


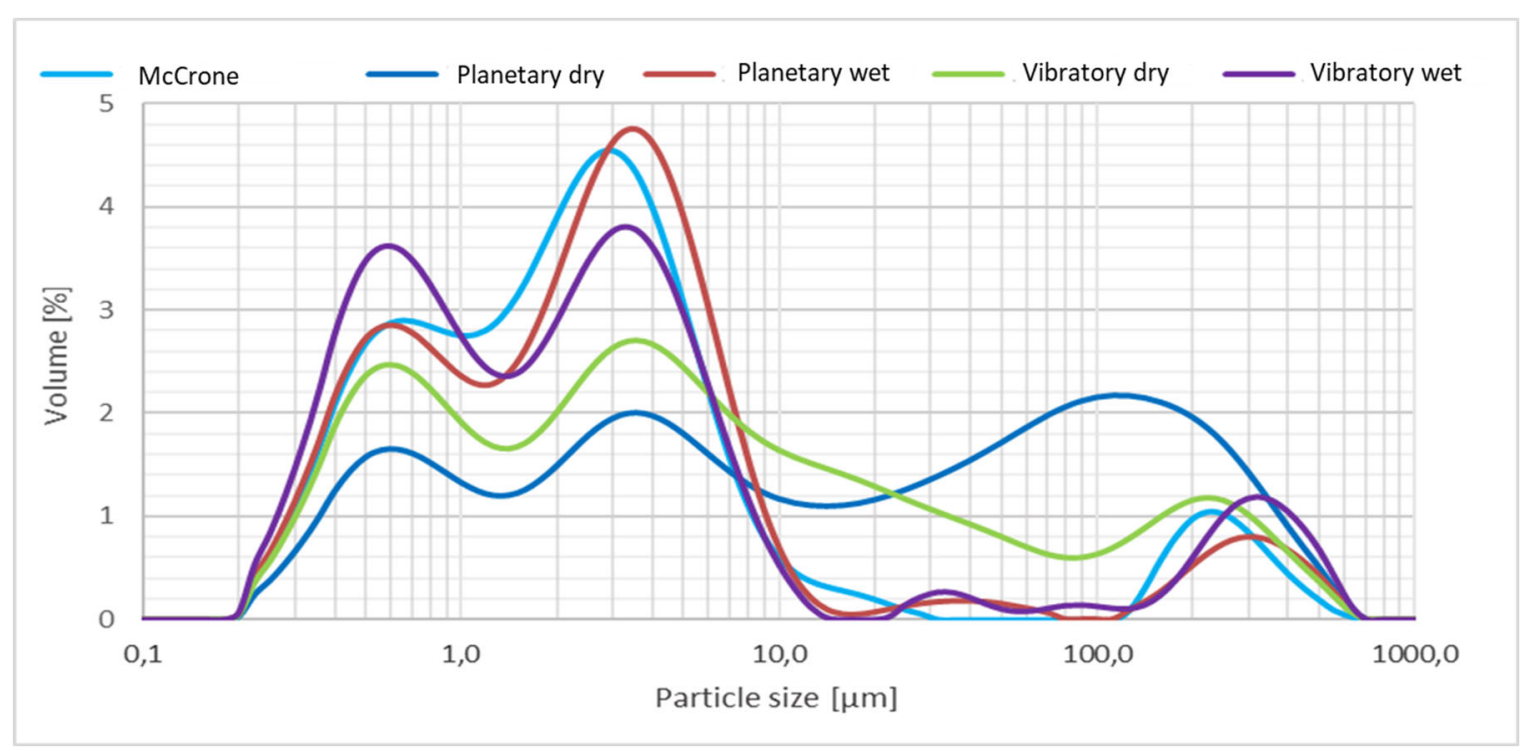

Figure 6. Particle distribution at 120 minutes

It is clearly shown that the agglomeration has occured in all types of laboratory mills, but this phenomenon is the most observed in dry condition of milling in the vibratory and planetary mill.

\section{Discussion}

Cubic tricalcium aluminate was prepared by modified Wesselsky-Jensen method. It was milled in a planetary mill to form an input specific surface area. The effect of milling in 3 types of laboratory mills on crystallinity a nd granulometry of tricalcium aluminate was monitored on the prepared mineral. Several methods have been considered for evaluating the effect of milling technology on the crystallization. The size of the crystallites was evaluated on 3 major crystallographic planes. To calculate the size of the crystallites, the simplest Scherrer method, subtracting the device extension, was chosen.

The device extension is determined because of the imperfection of the measuring device, which results in widening of the diffraction lines and thus negatively affects the resulting crystallite size values. The influence of device extension on the resulting crystallite size in the main crystallographic plane 440 was evaluated. The device extension can be considered to be constant up to the crystallite size of $2000 \AA$. Above $2000 \AA$, the device extension is almost twice, and above it significantly influences the resulting crystallite size. This fact, however, corresponds to the fact that the calculation of the crystallite size by the Scherrer equation is a method applicable only to a crystallite size of $200 \mathrm{~nm}$, which is $2000 \AA$, where the contribution of the device extension is constant.

The influence of the milling process on the clinker mineral crystallinity was evaluated by X-Ray analysis. The effect of the milling process is reflected in the diffraction pattern by a change in peak shape, reduction and extension. 1 minute of milling has almost no impact on the change in clinker crystallinity. After 120 minutes of milling, the most pronounced expansion and reduction of diffraction lines occurs, which corresponds to the decrease in the size of the crystallites. In the case of wet milling technology in a vibratory and planetary mill, the change in peak shape in the diffraction pattern is almost identical and insignificant compared with dry milling technology.

The milling time and selected milling technology have a major impact on the size of the crystallites. In general, it can be argued that milling in a dry condition has the most significant impact on the size of the crystallites. The liquid used in wet milling reduces the milling efficiency and thus the clinker mineral structure is not as damaged as in the case of dry milling. In the case of amorphization, dry milling in a planetary or vibratory mill is the most suitable.

Laser granulometry was used to compare the effect of milling technology on particle distribution and fineness. When comparing all three types of laboratory mills and the milling conditions, it can be stated that a large number of very fine particles is produced. However, during a long time of milling (120 minutes), very fine particles tend to form agglomerates with particle sizes above $100 \mu \mathrm{m}$. In wet condition of milling, agglomeration is somewhat suppressed by an inert liquid. Therefore, in the case of granulometry, the milling in the condition of isopropyl alcohol in the planetary and vibratory mill appears to be very effective. The efficiency of these two mills is essentially identical for the selected milling modes. Also, milling at McCrone mill seems to be effective in terms of fine particle size, but this type of mill is primarily designed for preparing samples for X-Ray analysis and not for larger laboratory milling. In the case of dry condition of milling in a planetary or vibratory mill, the largest amount of agglomerates is produced. This is due to the accumulation of a large amount of energy, that makes very fine particles which tend to agglomerate. 


\section{Conclusions}

The paper deals with the influence of milling technology on crystallographic and granulometric properties of synthetically prepared tricalcium aluminate. Dry milling in a planetary and vibratory mill has proven to have the greatest impact on these properties. With the dry milling technology in the mentioned types of mills, the size of the crystallites is significantly reduced. In terms of granulometry, the material is very effective and significantly refined. The problem arises with long grinding time, where the material tends to form agglomerates. By contrast, agglomeration is somewhat suppressed in wet condition of milling. In the case of selected grinding modes, the efficiency of grinding in the planetary and vibratory mill is approximately the same, both in terms of impact on structure and granulometry of the prepared $\mathrm{C}_{3} \mathrm{~A}$.

\section{Acknowledgements}

This work was financially supported by project number: FAST-J-19-5795: "Synthesis of Portland Clinker Phases".

\section{References}

Balaz, P. (2008). Mechanochemistry and nanoscience. In Mechanochemistry in nanoscience and minerals engineering (1 ${ }^{\text {st }}$ ed.). Berlin, Heidelberg: Springer-Verlag. https://doi.org/10.1007/978-3-540-74855-7_1

Duda, W. H. (1988). Cement data book (1 ${ }^{\text {st }}$ ed.). French \& European Publications.

Hewlett, P. (2003). Lea's chemistry of cement and concrete (4 ${ }^{\text {th }}$ ed.). Butterworth-Heinemann.

Maki, I. (1973). Nature of the prismatic dark interstitial phase in Portland cement clinker. Cement and Concrete Research, 3, 295313. https://doi.org/10.1016/0008-8846(73)90032-X

Ravaszová, S., \& Dvořák, K. (2018). Impact of milling process on the granulometric properties of triclinic alite. In IOP Conference Series: Materials Science and Engineering (vol. 385, conference 1). https://doi.org/10.1088/1757-899X/385/1/012044

Staněk, T. (2016). The influence of $\mathrm{SO}_{3}$ and $\mathrm{MgO}$ on kinetics of alite formation. Procedia Engineering, 151, 26-33. https://doi.org/10.1016/j.proeng.2016.07.353

Staněk, T., Sulovský, P., \& Boháč, M. (2017). Berlinite substitution in the cement clinker. Cement and Concrete Research, 92, 2128. https://doi.org/10.1016/j.cemconres.2016.11.007

Stephan, D., \& Wistuba, S. (2006). Crystal Structure refinement and hydration behaviour of doped tricalcium aluminate. Cement and Concrete Research, 36, 2011-2020. https://doi.org/10.1016/j.cemconres.2006.06.001

Taylor, H. F. W. (1997). Cement chemistry (2 ${ }^{\text {nd }}$ ed.). London: Thomas Telford Publishing. https://doi.org/10.1680/cc.25929

Wesselsky, A., \& Jensen, O. M. (2009). Synthesis of pure Portland cement phases. Cement and Concrete Research, 39 , 973-980. https://doi.org/10.1016/j.cemconres.2009.07.013 